\title{
Estabilidad y diseño de un controlador LQR para un sistema Bola-Viga
}

\section{Stability and design of a LQR controller for a Ball-Beam system}

\author{
TELLEZ-CUEVAS, Pedro†*, HERNÁNDEZ-LUNA, Aldo y CRUZ-LUNA, Manuel \\ Tecnológico Nacional de México/Instituto Tecnológico Superior de Huauchinango. \\ ID 1 ${ }^{\text {er }}$ Autor: Pedro, Tellez-Cuevas / ORC ID: 0000-0002-3235-1898, Researcher ID Thomson: G-2875-2019, CVU \\ CONACYT ID: 342839.
} ID $1^{\text {er }}$ Coautor: Aldo, Hernández-Luna / ORC ID: 0000-0002-7717-5314, Researcher ID Thomson: I-1165-2018, CVU
CONACYT ID: 441305.

ID $2^{\text {do }}$ Coautor: Manuel, Cruz-Luna / ORC ID: 0000-0002-0640-8926, Researcher ID Thomson: H-8709-2018, CVU CONACYT ID: 368159

DOI: $10.35429 /$ JID.2020.10.4.23.29

Recibido 23 de Marzo, 2020; Aceptado 12 de Junio, 2020

\section{Resumen}

Este articulo presenta el diseño de un controlador LQR para un sistema Bola-Viga, así como su análisis de estabilidad, el control de un sistema Bola-Viga es uno de los más interesantes para la ingeniería de control ya que es un sistema dinámico altamente no lineal. Los objetivos de este documento se centran en el rendimiento del sistema usando un control LQR para diferentes perturbaciones así mismo obtener los planos de fase. El trabajo parte con el modelado del sistema de bola-viga, el cual consiste de dos brazos mecánicos, una caja de engranes y un servomotor de $\mathrm{CD}$, posteriormente se diseña el control LQR, esto permite realizar la simulación y la obtención de la respuesta del controlador bajo diferentes condiciones. En el sistema el par de entrada se genera desde el servomotor de CD para controlar la posición de la bola en la viga, donde la bola rueda libremente sobre la viga. El análisis del rendimiento se lleva a cabo utilizando LQR robusto y se presentan las características de rendimiento del sistema. Finalmente, el análisis de estabilidad se lleva a cabo trazando los planos de fase.

Sistema de bola-viga, Espacio de estados, Análisis de estabilidad

\begin{abstract}
This article presents the design of an LQR controller for a Ball-Beam system, as well as its stability analysis, the control of a Ball-Beam system is one of the most interesting for control engineering since it is a highly non-dynamic system linear. The objectives of this document focus on the performance of the system using an LQR control for different disturbances as well as obtaining the phase plans. The work starts with the modeling of the ballgirder system, which consists of two mechanical arms, a gear box and a DC servomotor, later the LQR control is designed, this allows simulation and obtaining the response of the controller under different conditions. In the system the input torque is generated from the DC servo motor to control the position of the ball on the beam, where the ball rolls freely on the beam. Performance analysis is performed using robust LQR and the performance characteristics of the system are presented. Finally, the stability analysis is carried out by plotting the phase planes.
\end{abstract}

Ball and beam system, State space, Stability analysis

Citación: TELLEZ-CUEVAS, Pedro, HERNÁNDEZ-LUNA, Aldo y CRUZ-LUNA, Manuel. Estabilidad y diseño de un controlador LQR para un sistema Bola-Viga. Revista del Diseño Innovativo. 2020, 4-10: 23-29

\footnotetext{
*Correspondencia al Autor (Correo electrónico: ingelecptc@gmail.com)

$\dagger$ Investigador contribuyendo como primer autor.
} 


\section{Introducción}

La dinámica de un sistema está condicionado por acciones, ya sea de un modo deseado a través de la manipulación de variables o por perturbaciones que son aquellas variables no manipuladas. El desafío actual es el modelado y control, interrelacionados, de sistemas modernos y complejos, tales como el control de tráfico, procesos químicos y sistemas robóticos [1]. El control de un sistema Bola-Viga es uno de los más interesantes para la ingeniería de control ya que es un sistema dinámico altamente no lineal, el cual consiste en una viga que rota en donde una bola se mueve libremente cambiando de posición mientras rueda en la viga. Este sistema se puede aplicar en problemas de estabilidad para varios sistemas, como la estabilidad horizontal de un avión durante el aterrizaje y el flujo de aire turbulento, y el problema de equilibrio de los robots.

Una propiedad interesante del sistema de bola-viga que motivó la investigación es que, es un sistema inestable. Se han realizado muchos estudios sobre el sistema de bola-viga. Hirsch [2] construyó el sistema bola-viga, uso un sensor ultrasónico para medir la posición de la bola. El ángulo de la viga lo midió a través de un potenciómetro. El motor con caja de cambios se accionó con un circuito de amplificador operacional de alta potencia. Yu [3] usa un controlador PD para controlar el modelo no lineal del sistema bola-viga. El controlador aplicado es una síntesis de tipo PD que muestra la respuesta con un tiempo de establecimiento de aproximadamente $150 \mathrm{~ms}$. Sin embargo, existen algunos errores de estado estable.

Los objetivos de este documento se centran en el rendimiento del sistema usando un control LQR para diferentes perturbaciones así mismo obtener los planos de fase. La organización de este documento es la siguiente: de inicio se realiza el modelado del sistema de bola-viga, posteriormente se diseña el control LQR, esto permite la configuración experimental y la obtensión de resultados.

\section{Modelado del sistema de bola y viga}

El modelado es el primer paso en el diseño de un lazo de control. Cuanto mayor es el conocimiento del proceso, mejores son los resultados que obtendremos, ya que el diseño del control será basado en el modelo.
La configuración mecánica del sistema bola-viga se muestra en la figura 1 [4], consiste de dos brazos mecánicos, una caja de engranes y un servomotor de CD. La bola se coloca en la viga, donde rueda libremente a lo largo de su plano horizontal. El brazo de palanca está unido a la viga en un extremo y el servo engranaje en el otro.

Aplicando la ley de Kirchhoff de voltajes al motor de $\mathrm{CD}$ se obtiene

$$
V_{\text {in }}=I_{m} R_{m}+K_{b} \dot{\theta}+L_{m} \frac{d i}{d t}
$$

Donde $\dot{\theta}$ es la velocidad angular, $V_{\text {in }}$ es el voltaje de entrada, $I_{m}, R_{m}$ y $L_{m}$ son la corriente, la resistencia y la inductancia de armadura respectivamente y $K_{b}$ la constante de la fuerza electromotriz. Si la inductancia de armadura tiene un valor muy pequeño la ecuación (1) se reduce a:

$V_{\text {in }}=I_{m} R_{m}+K_{b} \dot{\theta}$

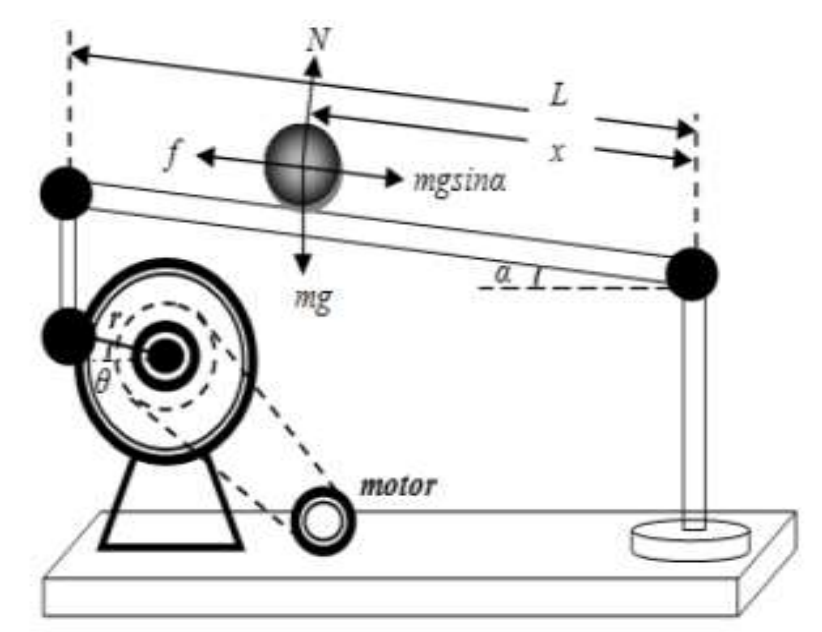

Figura 1 Sistema bola viga

El par que actúa sobre la viga es:

$$
\tau_{m}=\frac{1}{K_{g}}\left(J_{m} \ddot{\theta}+B_{m} \dot{\theta}\right)
$$

El coeficiente de fricción viscosa es $B_{m}$, el par rotacional es $\tau_{m}, K_{g}$ es la relación entre la caja de engranes del motor y el servo, $J_{m}$ es la inercia. En base a las ecuaciones anteriores el modelo del motor queda descrito como

$V_{\text {in }}=\frac{R_{m} J_{m}}{K_{m} K_{g}} \ddot{\theta}+\left(K_{b}+\frac{R_{m} B_{m}}{K_{m} K_{g}}\right) \dot{\theta}$ 
Para obtener el modelo matemático del sistema bola viga utilizamos las ecuaciones de Euler-Lagrange, considerando que el sistema no tiene perturbaciones y no hay fricción quedando

$H=T-P$

$\left.H=\frac{1}{2}\left[U_{1}+m r^{2}\right) \dot{\alpha}^{2}+\frac{7}{5} m \dot{r}^{2}\right]-\left(m g r+\frac{L}{2} M g\right) \sin \alpha$

El lagrangiano $\mathrm{H}$ resume la dinámica del sistema. Puesto que no hay fuerza externa sobre la bola, las ecuaciones de movimiento de Lagrange se dan en las ecuaciones (7) y (8)

$\left(J_{1}+m r^{2}\right) \ddot{\alpha}+2 m r \dot{r} \dot{\alpha}+\left(m g r+\frac{L}{2} M g\right) \cos \alpha=\tau$

$\frac{7}{5} \ddot{r}-r \dot{\alpha}^{2}+g \sin \alpha=0$

Considerando que sin fricción el ángulo de la viga conduce a que $g \sin \alpha \approx g \alpha$, entonces al linealizarse estas ecuaciones se puede estimar cuando sistema se aproxima a un punto de equilibrio. En este punto $\dot{\alpha} \approx 0$ entonces $-r \dot{\alpha}^{2} \approx 0$ y las ecuaciones (7) y (8) quedan

$\left(m r^{2}+K_{1}\right) \ddot{\alpha}+\left(2 m r \dot{r}+K_{2}\right) \alpha+\left(m g r+\frac{L}{2} M g\right) \cos \alpha=\tau$

$K_{4} \ddot{r}-r \dot{\alpha}^{2}+g \alpha=0$

Donde

$K_{1}=\frac{R_{m} J_{m} L}{K_{m} K_{g} d}+J_{1}$

$K_{2}=\frac{L}{d}\left(\frac{K_{m} K_{b}}{R_{m}}+K_{b}+\frac{R_{m} B_{m}}{K_{m} K_{g}}\right)$

$K_{3}=1+\frac{K_{m}}{R_{m}}$

$K_{4}=\frac{7}{5}$ estados [5]

La representación en el espacio de

$\dot{x_{1}}=x_{2}$

$\dot{x_{2}}=-\frac{g}{K_{4}} x_{3}$

$\dot{x_{3}}=x_{4}$

$$
\begin{aligned}
& \dot{x_{4}}=\frac{1}{m x_{1}{ }^{2}+K_{1}}\left[K_{3} V_{i n}-\right. \\
& \left(2 m x_{1} x_{2}-K_{2}\right) x_{4}-\left(m g x_{1}+\right. \\
& \left.\left.\frac{L}{2} M g\right) \cos x_{3}\right]
\end{aligned}
$$

La variable $x_{1}=r(m)$ es la posición de la bola en la viga, $x_{2}=\dot{r}(\mathrm{~m} / \mathrm{s})$ representa la velocidad de la bola, $x_{3}=\alpha(\mathrm{rad})$ indica la posición angular de la viga y $x_{4}=\dot{\alpha}(\mathrm{rad} / \mathrm{s})$ es la velocidad de la viga.

El modelo representado por las ecuaciones diferenciales de estado es no lineal como la mayoría de los sistemas físicos. Este modelo se puede aproximar a su versión lineal bajo ciertas condiciones en determinados puntos de equilibrio. La linealización se realiza alrededor de un punto o trayectoria de operación, definido por valores nominales.

$$
\text { El punto } x_{e q}=f\left(x_{e q}, 0\right)=0
$$

$$
\begin{aligned}
& 0=x_{2} \\
& 0=-\frac{g}{K_{4}} x_{3} \\
& 0=x_{4} \\
& 0=\frac{1}{m x_{1}^{2}+K_{1}}\left[K_{3} V_{i n}-\left(2 m x_{1} x_{2}-K_{2}\right) x_{4}-\right. \\
& \left.\left(m g x_{1}+\frac{L}{2} M g\right) \cos x_{3}\right]
\end{aligned}
$$

Despejando, obtenemos que el punto de equilibrio resulta

$x_{e q}=\left[\begin{array}{llll}-\frac{L M}{2 m} & 0 & 0 & 0\end{array}\right]$

Tomando el punto de equilibrio se linealiza el sistema alrededor de dicho punto derivando las ecuaciones de estado para obtener las matrices A y B por lo que se obtiene a partir de:

$$
A=\left.\frac{\partial f}{\partial x}\right|_{x=x_{e q}} \mathrm{y} \quad B=\left.\frac{\partial f}{\partial u}\right|_{x=x_{e q}}
$$

Para este trabajo se consideran los siguientes datos del sistema $\mathrm{L}=0.7 \mathrm{~m}$; $\mathrm{M}=18.848 \times 10^{-3} \mathrm{Kg} ; \mathrm{m}=0.5 \mathrm{Kg} ; \mathrm{g}=9.81 \mathrm{~m} / \mathrm{s}^{2}$; $\mathrm{J}_{1}=7.35 \times 10^{-4} \mathrm{Kg} \cdot \mathrm{m}^{2} ; \mathrm{d}=0.075 \mathrm{~m} ; \mathrm{B}_{\mathrm{m}}=0.4 \times 10^{-6}$ N.m.s; $K_{b}=0.022 \mathrm{~V} / \mathrm{rad} / \mathrm{s} ; \mathrm{K}_{\mathrm{m}}=0.022 \mathrm{~N} \mathrm{~m} / \mathrm{A}$; $\mathrm{R}_{\mathrm{m}}=9.4 \Omega ; \tau=0.0163$ N.m; $\mathrm{J}_{\mathrm{m}}=8.5 \times 10^{-7} \mathrm{Kg} . \mathrm{m}^{2}$; $\mathrm{I}_{\mathrm{m}}=0.76 \mathrm{~A}$; 
(12) quedando

Ya con los datos se sustituyen en (11) y

$$
\begin{aligned}
& A=\left[\begin{array}{cccc}
0 & 1 & 0 & 0 \\
0 & 0 & -7.0014 & 0 \\
0 & 0 & 0 & 1 \\
-1140.9 & 0 & 0 & -48.25
\end{array}\right] \\
& B=\left[\begin{array}{c}
0 \\
0 \\
0 \\
233.148
\end{array}\right]
\end{aligned}
$$

\section{Diseño del controlador LQR.}

El control LQR es un controlador que proporciona el mejor rendimiento posible, ofrece una ley de control simple, muy robusto tanto en tiempo continuo o en tiempo discreto. Para el control óptimo, las especificaciones de control son formuladas en una función de costo. La función de costo (también conocida como figura de mérito, índice de desempeño, etc.), es una función que penaliza el "mal" comportamiento del sistema, es decir cuánto más lejos este él sistema de la situación deseada, mayor será el valor de la función de costo. Entonces, el objetivo del controlador óptimo, será minimizar esta función.

El control LQR se plantea como la necesidad de calcular la mejor entrada $u(t)$, que permita llevar el sistema de un estado inicial $x\left(t_{o}\right)$, a un estado final $x\left(t_{f}\right)$ en un tiempo $t_{f}-$ $t_{o}$. Para calcular la ganancia de realimentación $\mathrm{K}$ es siguiendo el criterio de optimización minimizando el funcional cuadrático

$J=\int_{0}^{t_{\infty}}\left[x^{T}(t)+Q x(t)+\right.$ $\left.u^{T}(t) R u(t)\right] d t$

Donde $\mathrm{Q}$ y $\mathrm{R}$ son matrices positivas definidas, generalmente diagonales o cuando menos simétricas, que determinan la importancia de cada parámetro dentro de la función de costo, que ponderan las variables de estado y la actuación respectivamente, la matriz Q la importancia de los estados durante la transición y R la importancia de la entrada.

La formulación del problema utilizando una matriz $\mathrm{R}$ distinta de cero, tiene particular importancia en la práctica ya que esta matriz nos limitará el valor de la entrada u, aplicada al sistema.
Como el criterio se basa en funcionales cuadráticos, recibe el nombre de regulador lineal cuadrático (LQR). El funcional $\mathrm{J}$ puede interpretarse como el costo total de la transición de las variables de estado de un punto a otro.

Con las siguientes restricciones

$$
\begin{aligned}
& \dot{x}=A x(t)+B u(t) \\
& u(t)=-K x(t)
\end{aligned}
$$

No hay reglas que puedan emplearse de forma general para la selección de las matrices Q y R. Una regla simple consiste en escoger Q y $\mathrm{R}$ diagonales, asignando valores grandes a aquellas variables que se desee minimizar.

En cualquier caso, los valores de Q y R deben ser siempre positivos o cero. El conocimiento que se posea del sistema resulta fundamental en la selección de estas matrices. Aun así, es conveniente calcular diferentes controladores, en base a distintos valores para las matrices $\mathrm{Q}$ y $\mathrm{R}$, y verificar su efectividad mediante la simulación. Una ventaja importante del control LQR es que, sea cual sea la elección de las matrices Q y R, se preserva la estabilidad asintótica y la robustez del controlador.

Considerando lo anterior, se definen las matrices $\mathrm{Q}$ y $\mathrm{R}$, con ellas se determina la importancia relativa del error y el gasto de energía de la señal de control por tanto:

$$
\begin{aligned}
& Q=\left[\begin{array}{cccc}
50000 & 0 & 0 & 0 \\
0 & 50000 & 0 & 0 \\
0 & 0 & 5000 & 0 \\
0 & 0 & 0 & 5
\end{array}\right] \\
& R=[0]
\end{aligned}
$$

\section{Simulacion y resultados}

La simulación del sistema bola-viga con controlador se desarrolla sobre el software MATLAB/SIMULINK. El obtener la matriz K, analíticamente no es tarea fácil, MATLAB cuenta con la función $K=\operatorname{lqr}(\mathrm{A}, \mathrm{B}, \mathrm{Q}, \mathrm{R})$. Para ello es necesario ajustar $Q$ y $R$ hasta obtener el desempeño deseado. 
La matriz A y B se toman de (13) y (14) y la matriz Q y R de (18) y (20) respectivamente con lo que se obtuvo:

$$
K=\left[\begin{array}{llll}
-228.553 & -239.47 & 114.978 & 2.248
\end{array}\right]
$$

La Figura 2 muestra el diagrama de control del sistema bola-viga en SIMULINK con el controlador LQR.

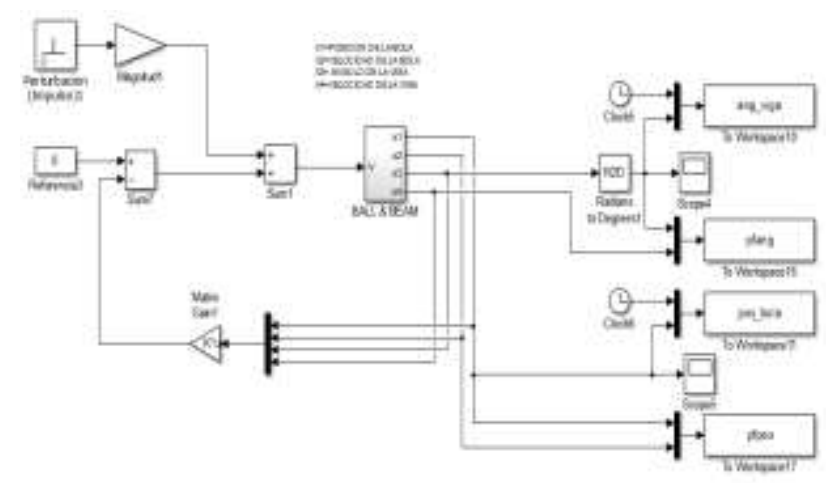

Figura 2 Sistema de control LQR en SIMULINK

Los resultados de la simulación para el control de seguimiento se ilustran en la Gráfico 1 para el caso de una perturbación impulso cuya magnitud es de $5 \mathrm{~N}$ con una referencia $0^{\circ}$. La respuesta que se grafica corresponde a la posición angular de la viga con respecto a la horizontal, se observa que tiene dos sobreelongaciones una de $\pm 1.7^{\circ}$ y un tiempo de asentamiento de $3 \mathrm{~s}$.

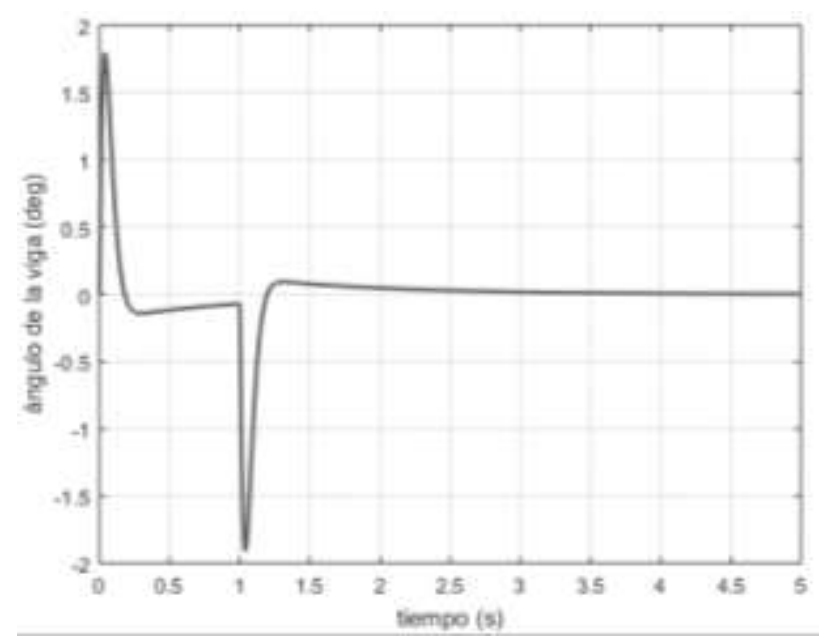

Gráfico 1 Respuesta del sistema con una perturbación de $5 \mathrm{~N}$.

Los Gráficos 2 y 3 muestran el comportamiento del sistema con unas perturbaciones de $25 \mathrm{~N}$ y $50 \mathrm{~N}$ respectivamente en donde se observa que la salida sigue con éxito la trayectoria de referencia, proporcionan un buen rendimiento ambas con un tiempo de asentamiento para el caso de ambas figuras de 3 s.
Pero en el caso de la respuesta del Gráfico 1 se tienen sobreenlongaciones de $8^{\circ},-12^{\circ}$ y dos pequeños picos de $\pm 1^{\circ}$. La respuesta de la Gráfico 2 muestra que alcanza unas sobreelongaciones de $15^{\circ}, 12^{\circ}, 4^{\circ}, 1^{\circ},-20^{\circ},-6^{\circ}-0.5^{\circ}$, hasta que alcanza el valor de referencia.

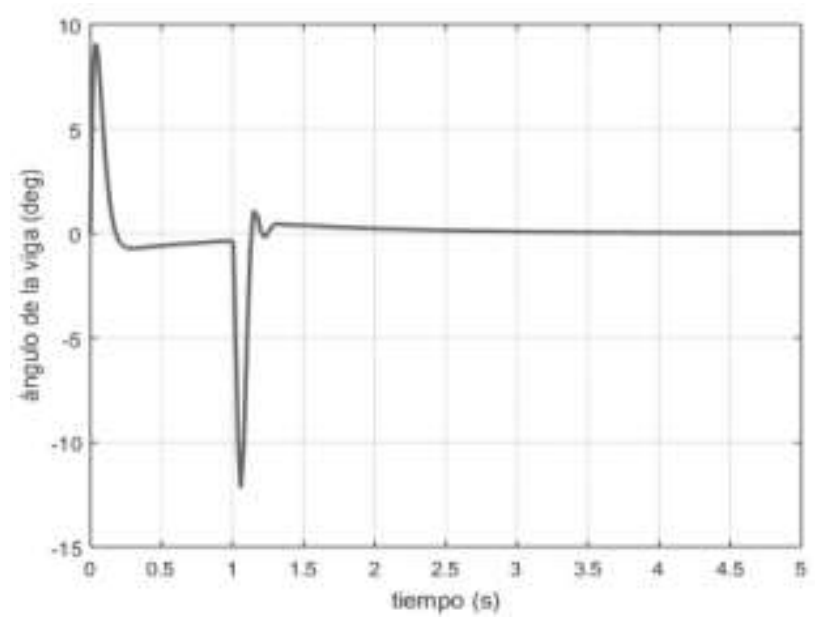

Gráfico 2 Respuesta del sistema con una perturbación de $25 \mathrm{~N}$.

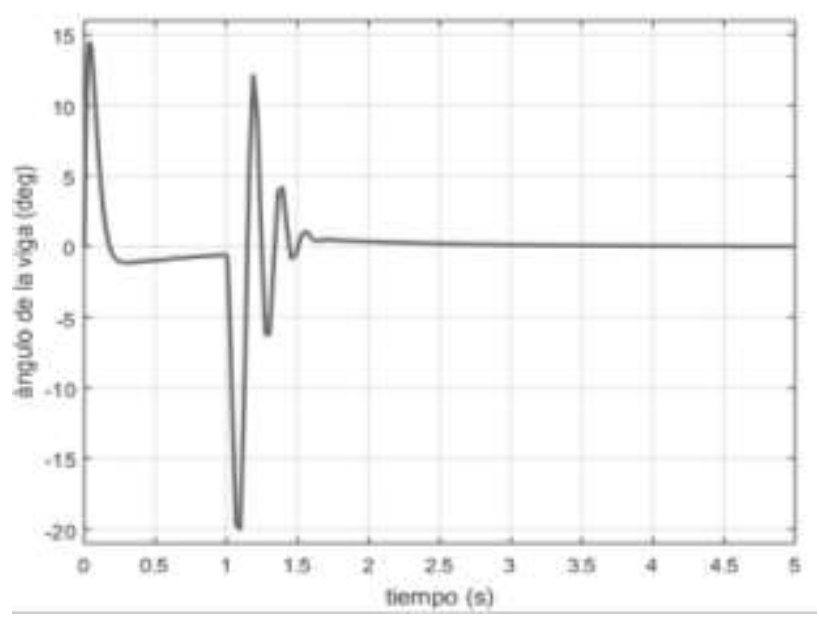

Gráfico 3. Respuesta del sistema con una perturbación de $50 \mathrm{~N}$.

La estabilidad es una característica importante a considerar en el desarrollo y análisis de un controlador, cuando el sistema es lineal se dispone de algunos criterios, entre los cuales está el criterio de estabilidad de Routh o el Nyquist, sin embargo cuando el sistema es no lineal estos criterios no son aplicables [6].

Para verificar la estabilidad del sistema por medio del análisis gráfico con el plano de fase en el que se grafica la posición angular (eje x) contra la velocidad angular (eje y) de la respuesta del sistema. 
El Gráfico 4, muestra la trayectoria en el plano de fase del sistema, en ella se observa que la trayectoria parte desde un punto establecido por las condiciones iniciales, que en este caso son cero, y después de cierto tiempo llega al punto esperado ya que se ha perturbado con un impulso de $5 \mathrm{~N}$.

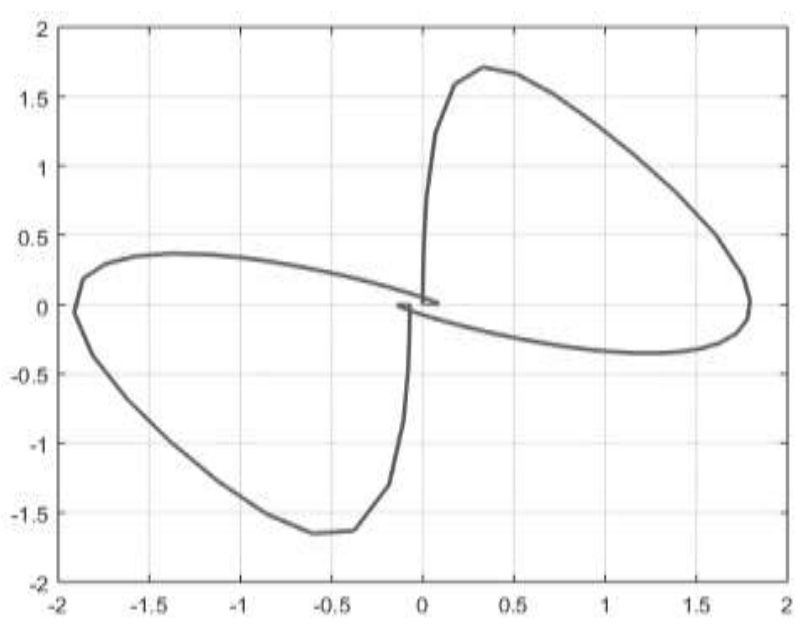

Gráfico 4 Plano de fase del sistema para una perturbación de $5 \mathrm{~N}$

En el Gráfico 5 se observa que la trayectoria es muy parecida a las del Gráfico 4, solo con pequeñas variaciones en su trayectoria.

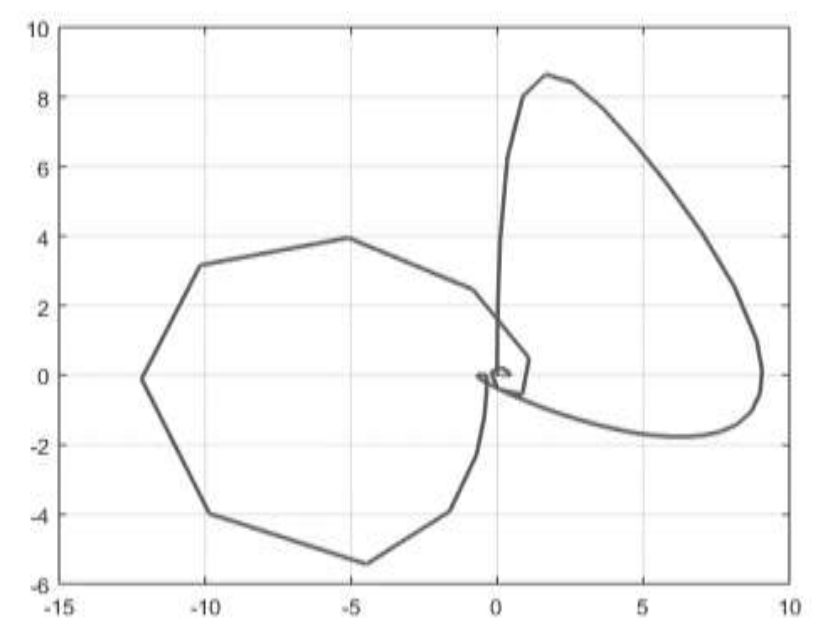

Gráfico 5 Plano de fase del sistema para una perturbación de $25 \mathrm{~N}$

El Gráfico 6 describe una trayectoria más irregular debido a las sobreelongaciones, que se observan en el Grafico 3, pero alcanza el punto deseado, en ambas trayectorias para las diferentes perturbaciones las respuestas convergen al mismo valor de referencia.

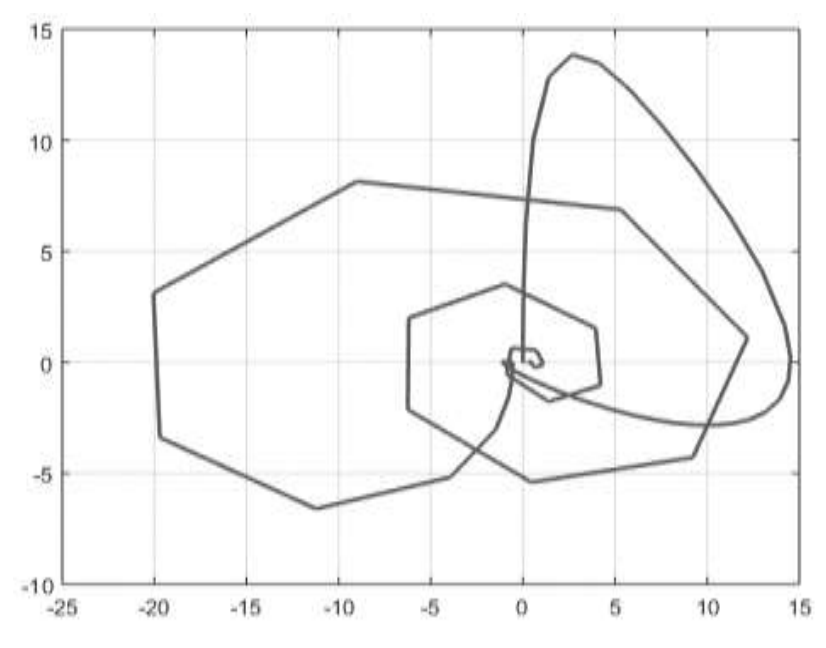

Gráfico 6 Plano de fase del sistema para una perturbación de $50 \mathrm{~N}$

\section{Agradecimiento}

Los autores desean expresar su agradecimiento a las carreras de Ingeniería Eléctrica e Ingeniería Informática del Instituto Tecnológico Superior de Huauchinango por el apoyo y las facilidades para el desarrollo de este trabajo.

\section{Conclusiones}

En el presente trabajo se desarrolla un controlador LQR para un sistema bola viga, el cual es representado por un modelo no lineal.

Se obtiene el comportamiento dinámico del sistema con una referencia de 0 y diferentes perturbaciones impulsos, la respuesta obtenida muestra que el sistema responde rápido con pequeñas sobreelongaciones para perturbaciones magnitudes pequeñas y en caso de magnitudes grandes tiene picos mayores con respecto con los de menor magnitud pero en ambos caso alcanza el valor deseado.

Para la estabilidad por medio de plano de fase, las trayectorias de la respuesta del sistema no lineal con el controlador LQR, las respuestas convergen al mismo valor de referencia. Finalmente, este estudio proporciona un rendimiento dinámico con precisión de la posición angular de la viga.

\section{Referencias}

[1] Dorf, Richard C.y Bishop Robert H., Sistemas de Control Moderno, 10. Ed., Prentice-Hall, 2007.

[2] Hirsch, R. (1999). Mechatronic instructional systems ball on beam system. Shandor Motion Systems. 
[3] Yu, W.; and Ortiz, F. (2005). Stability analysis of PD regulation for ball and beam system. IEEE Conference on Control Application, 517-522.

[4] B. M. Hung, S. S. You, H. S. Kim, T. W. Lim. "Embedded controller building for ball and beam system using optimal control synthesis", Journal of Engineering Science and Technology Vol. 12, No. 6 (2017) 1460 -1474 .

[5] Keshmiri, M.; Jahromi, A.F.; Mohebbi, A.; Amoozgar, M.H.; and Xie, F.W. (2012). Modeling and control of ball and beam system using model based and non-model based control approaches. International Journal on Smart Sensing and Intelligent Systems, 5(1), 14-35.

[6] Leonid Reznik, "Fuzzy Controllers", Newnes, Butterworth-Heinemann Linacre House, Jordan Hill, Oxford OX2 8DP, 1er Ed,, ISBN 075063429 4., 1997. 